Communication

\title{
A Brief Neurocognitive Assessment for HAND in Low Resourced Occupational Health Settings in Developing Countries
}

\author{
Charles Van Wijk \\ Private Practice, Simon's Town, South Africa \\ Email address: \\ chvanwij@gmail.com
}

\section{To cite this article:}

Charles Van Wijk. A Brief Neurocognitive Assessment for HAND in Low Resourced Occupational Health Settings in Developing Countries. Central African Journal of Public Health. Vol. 5, No. 1, 2019, pp. 24-30. doi: 10.11648/j.cajph.20190501.14

Received: November 15, 2018; Accepted: December 6, 2018; Published: January 24, 2019

\begin{abstract}
HIV associated neurocognitive disorder (HAND) is highly prevalent in sub-Saharan Africa, and with the support of anti-retroviral treatment, more people are able to remain in the workplace. However, the potential for HAND to affect the individual health and safety of employees is substantial. Formal assessment of HAND is resource intensive, and often outside the capability of smaller organisations which offers occupational health support. This paper describes a protocol for a brief neurocognitive assessment for HAND in low resourced occupational health settings. The paper firstly describes the development - following seven guidelines - of a relatively inexpensive and relatively brief protocol to assess for the presence of HAND, in a South African low resourced occupational health setting. The resultant Brief Neurocognitive Assessment (BNCA) is then described in detail, as well as the larger process within which it is administered. Further, neuropsychological data from the first 60 completed assessments are presented, together with a discussion of its adherence to the guidelines set out prior to its development. A number of neuropsychological markers appeared to differentiate between the normal and mild impairment groups, suggesting that the BNCA may be useful in identifying individuals with potential problematic neurocognitive functioning. A brief assessment such as the BNCA could provide meaningful recommendations regarding both clinical management and workplace utilisation to employers. It would be important that proper processes (e.g. conditions for assessments, appropriate follow up procedures, confidentiality, and so forth) are imposed and maintained. Finally, this paper invite discussion for improved management of HAND assessment in the workplace.
\end{abstract}

Keywords: HIV Associated Neurocognitive Disorders, Occupational Health and Safety, Workplace Health

\section{Introduction}

This paper aims to describe a protocol for a brief neurocognitive assessment for HIV-associated neurocognitive disorder (HAND) in low resourced occupational health settings. It also presents some preliminary data for the protocol, in order to invite discussion for improved management of HAND screening.

\subsection{Reported Prevalence of HAND}

HAND status is currently determined using the Frascati criteria, which requires neuropsychological (NP) scores across seven domains to be compared to normative data using standard deviations as indicators of impairment [1]. This model provides for three progressive conditions of HIV-associated neurocognitive decline, namely asymptomatic neurocognitive impairment (ANI), mild neurocognitive disorder (MND) and HIV-associated dementia (HAD).

HAND remains highly prevalent, with global prevalence reported from $30-50 \%$ in people living with HIV and AIDS (PLWHA), depending on disease stage and the use of anti-retroviral treatment (ART). Despite the increased availability of ART, HAND remains common in both the developed and developing world, with most cases being ANI or MND [2-8]. In sub-Saharan Africa (SSA), where there is high prevalence of HIV itself, HAND prevalence rates ranging from $39-76 \%$ have been reported for general clinical 
populations [6, 9]. Prevalence in the active workforce is more difficult to estimate. A small workplace study from South Africa (SA) suggested $32 \%$ of PLWHA could be classified with milder forms of HAND [10].

\subsection{Neuropsychological Effects of HAND}

Although there is evidence of wide variability in deficits associated with HIV [11], most studies report a fairly typical pattern of NP impairment [4, 12-14] relating to:

a) Motor slowing;

b) Speed of Information Processing;

c) Learning \& memory (e.g. verbal episodic memory);

d) Attention \& working memory;

e) Executive functions.

Visuo-perception and speech \& language appear to be spared more often.

Impairment of these domains may have significant implications for workplace health and safety, and it is in the workplace where milder forms of HAND are more likely to be encountered [15]. In the case of SA, the increasing availability of ART has allowed more PLWHA to remain in the workplace [16], and together with greater awareness of the neurocognitive symptoms associated with HIV, have raised new concerns around safety-critical tasks requiring intact cognitive functioning. For example, HIV associated neurocognitively impaired individuals are at increased risk for impaired driving performance [17], and even with only mild impairments, are five times more likely to report diminished work ability [15]. Workplace safety concerns become particularly pertinent in cases where neurocognitive decline precedes physical symptoms. With the onus on employers to support the health of employees, as well as their safety in the workplace, many employers now offer HIV care through workplace outreach programs.

\subsection{Identification of $H A N D$}

Formal NP assessment remains the gold standard for the classification of HAND [12, 18]. However, it is resource intensive, and poses challenges in poorly resourced settings, for example in SA where smaller employers are motivated to provide HIV/AIDS support, but do not have the resources often found within larger private organisations or academic institutions.

In the workplace there is a need for a NP test battery to identify HAND, in order to guide decisions both for clinical treatment purposes, and for the workplace safety of individuals. Such a requirement is associated with three challenges (among others) that create obstacles for the appropriate support to PLWHA employed in poorer resourced workplaces, namely:

a) It requires professionals with NP expertise to do the assessments, which has significant cost implications in terms of the time required for administration, scoring, and interpretation of tests; b) Commercially available assessment instruments carries substantial costs (including initial capital costs, and often further per test cost in the form of response booklets, etc.), which may become prohibitive for smaller companies;

c) There is a dearth of normative data against which to interpret NP performance in specific contexts [13, 19], which in turn may have significant implications for personal career prospects as well as workplace safety in general.

\section{HAND Assessment Protocol}

This paper describes an attempt in a SA low resourced occupational health setting to develop a relatively inexpensive and relatively brief protocol with which to meaningfully assess for the presence of HAND. When the protocol was considered, a number of guidelines were set to direct the composition of the battery:

1. It had to be brief (to limit the time required for administering and scoring), but simultaneously comprehensive enough to enable meaningful diagnostic classification (e.g. according to the Fascati criteria).

2. It needed to be inexpensive to obtain, and inexpensive to maintain. Thus the preference was for NP tasks/test available in the public domain, or reasonably available commercially (i.e. at low cost). Further, it should allow for repeated use (i.e. no per-test costs), resulting in a low 'per person' cost for each battery.

3. It needed to be mobile. Preference was for pen-and-pencil type tests, to limit reliance on testing 'hardware'. This would make it less expensive, but importantly to also make it easily transportable.

4. It needed to have some form of appropriate normative data available (e.g. from local SSA studies, or other comparable samples).

5. A history of use within the field of HAND assessment would be preferable. Tests needed to have a track record for clinicians to draw on the experience of others when interpreting results, supported by evidence of its usefulness in HAND assessment.

6. It needed to be language and culturally sensitive, as it would be used in multi-cultural workplaces, and by test administrators who may have different cultural origins from the testees. Language sensitivity was important, as, for example in SA, English is often used as common language, even when it may not the native language of either the tester or testee. Tests/tasks thus had to accommodate people with English as second or third language.

7. The final battery also needed to be simple enough that trained technicians could eventually administer it independently.

After an extensive review of the literature, a neurocognitive assessment process comprising three broad phases were put together (see Figure 1), referred to here as the 'Brief Neurocognitive Assessment' (BNCA). 


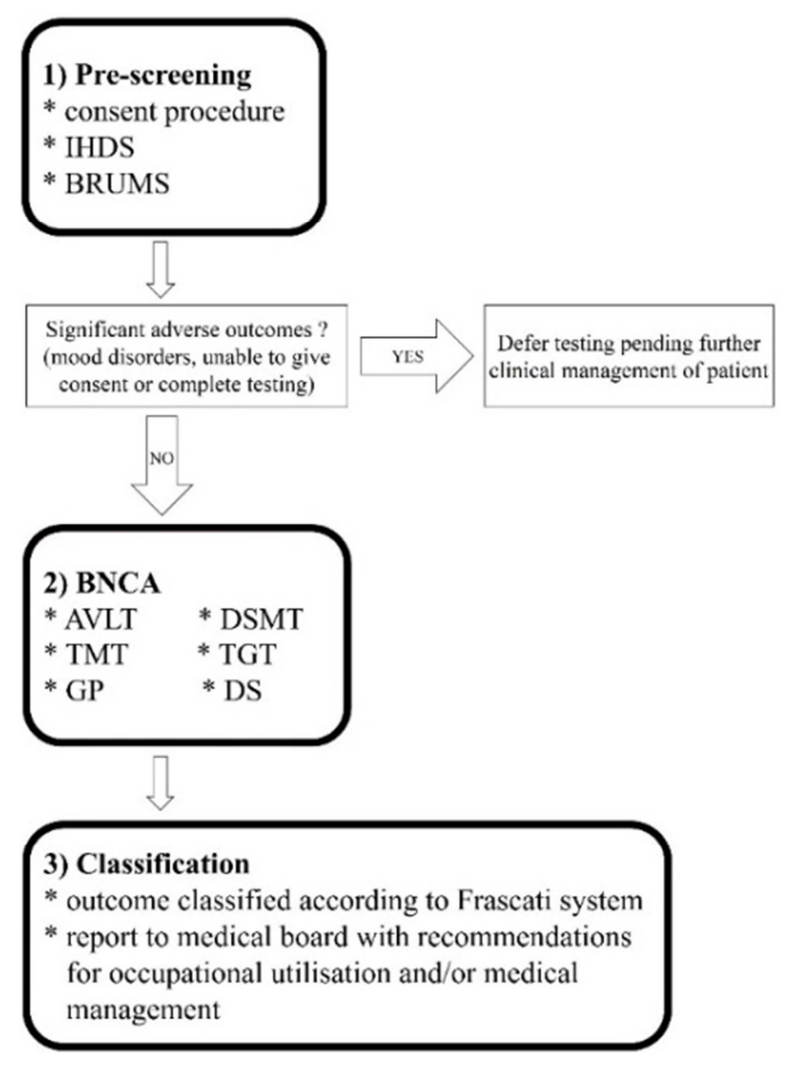

Figure 1. Three phases of the Brief Neurocognitive Assessment process.

\subsection{Pre-Screening}

1. Consent procedure. Firstly, Information \& Consent forms are completed. Persons who are neuropsychologically compromised may not be able to give appropriate consent, in which case testing is deferred pending further clinical management. If a person is able to give voluntary consent, they proceed to the Brunel Mood Scale (BRUMS).

2. BRUMS. Common mental disorders are highly prevalent among PLWHA in SA (38\%), with depression common in up to $25 \%$ of PLWHA during the course of the illness [20-21]. The BRUMS is not a clinical scale, but has an easy vocabulary, and is easy for candidates to use and understand during routine screening. If significant symptoms are reported, then testing is deferred pending further clinical management. Otherwise the person proceeds to do the IHDS. The BRUMS incidentally also appears to be a helpful indicator of English proficiency, prior to formal assessment.

3. IHDS. The International HIV Dementia Scale (IHDS) is widely used in cross-cultural screening studies [22], and its usefulness to screen for HAD in SA settings has been well established [23-25]. It further requires no special instrumentation, only a trained administrator and stopwatch. It is used here to screen for the presence of severe HAND. If severe impairment is suspected, then testing is deferred and referrals arranged for either further clinical management, or where indicated, for more comprehensive NP assessment.

\subsection{BNCA Battery}

Table 1 presents the test battery according to the domains tested. Total administration time is \pm 45 minutes, depending on the neurocognitive status of the testee.

Although there are a number of batteries available, various cross-cultural studies, and particular in Africa and India, indicated the centrality of the following assessment components for HAND assessment [6, 26-29], which is also recommended by the WHO. It is presented here in the order of administration during the BNCA.

WHO-UCLA Auditory Verbal Learning Test [30]. The AVLT taps verbal learning and memory. It was developed in the HIV field, specifically for individuals who do not have English as first language. Similar to other verbal learning tests, it scores the total number of words learned across five trials, as well as recall after an interference trial. There is a trial for delayed recall (administered after \pm 30 minutes), and a final recognition trial. Norms from SSA have been reported from Uganda [6, 28], Botswana [31] and South Africa [32]. There is no per-test cost involved.

The Trail-making Test [33]. The TMT tests visual attention and task switching, and requires testees to 'connect-the-dots' of 25 consecutive targets on a sheet of paper. The time taken to complete the test is used as the primary performance metric. On TMT-A the targets are all numbers, and the task is believed to tap attention and processing speed. On TMT-B testees have to alternate between numbers and letters, and is used as a measure for executive functioning. Limited literacy is required for this task, which was not considered problematic in the context where the BNCA was developed. Local SA norms have been reported [24, 34]. There is no per-test cost involved, and it only requires a pencil, paper, and stopwatch.

Table 1. Brief Neurocognitive Assessment battery.

\begin{tabular}{lll}
\hline NP Domain & Test / task & Abbreviation \\
\hline \multirow{2}{*}{ Verbal language } & Interview & BRUMS \\
& Brunel Mood Scale & DS-F \\
Attention / working memory & WAIS Digit Span & DS-B \\
Executive functions & (forward and backward) & TMT-B \\
Leaning & Trail-making Test (Trail B) & AVLT \\
Memory & WHO-UCLA Auditory Verbal Learning Test (trials 1-5) & AVLT \\
& AVLT & TMT-A \\
Speed of information processing & (trails 7-9) & DSMT \\
& Trail-making Test (Trail A) & GP-DH \\
Sensory-perceptual / motor skills & WAIS Digit Symbol Modalities Test & GP-NDH \\
& Grooved Pegboard & TGT \\
\hline
\end{tabular}


Grooved Pegboard (DH and NDH) [35]. The GP is a test of manipulative dexterity. Testees are asked to place 25 keyed pegs into an array of 25 slotted holes as fast as possible, using the designated hand. Their completion time is the score. Both hands are tested separately, in the order dominant hand and then non-dominant hand. Local South African norms are available [36], as well as data for other SSA samples [6, 28, 37]. The test (pegboard) needs to be purchased, but afterwards can be reused without incurring further cost.

Digit-Symbol Modalities Test [38]. The DSMT-coding is a pen-and-paper test of processing speed. Testees use a key of digit-symbol pairs at the top of the test page to fill in the correct symbol for each number as quickly as possible within a 120 -second time limit. The score is the number of correct items completed within the time limit. Although US or UK norms are available in the WAIS manual, other research papers report data that may be more locally appropriate, e.g. from Uganda [6, 28] and Botswana [31]. The initial WAIS involves a substantial capital outlay, although the per-test costs thereafter is relatively inexpensive.

Timed Gait Test [39-40]. The TGT is a test of gross motor function, with the purpose to measure psychomotor speed. Candidates have to walk 10-yards and return, over three trials, and the averaged time is use to indicate risk for HAND. Normative data is provided by Robertson et al. [40]. SSA data is also available for Botswana [37] and Uganda [6, 28]. The task incurs no costs.

Digit Span forward \& backward [38]. The DS is a test of attention and working memory, in which testees are given a series of digits verbally, and must repeat them in either the same or reverse order, with increasing difficulty. Although US or UK norms are available in the WAIS manual, other research papers report data that may be more locally appropriate, e.g. from Uganda [28] and South Africa [24]. The initial WAIS involves a substantial capital outlay, although the per-test costs thereafter is relatively inexpensive.

\subsection{Determining Outcome}

Performance scores are used to determine HAND status according to the Frascati classification system [1], and in the review below were allocated to each case after consensus between at least two psychologists. Outcomes in terms of work recommendations are forwarded to the employer organisation with the permission of the employees. Recommendations focusses on occupational utilisation and/or medical management.

\section{Review of First 60 Cases}

The first 60 consecutive BNCA's were reviewed as part of a clinical audit, and the preliminary data presented here. It comes from a SA organisation that provides internal occupational health-care to employees and their families. The study was conducted according the principles set out in the Declaration of Helsinki (2013), and the appropriate ethical approval had been obtained.

\subsection{Participants}

All 60 were HIV positive (time since diagnosis 1-10 years), and $90 \%$ were on ART. Despite variable health histories, all but 3 were physically healthy at the time of assessment, suggesting that their medical condition was well managed. None reported major psychiatric or neurological disorders. They had variable job descriptions, with different associated cognitive demands, and all considered as skilled labour. All had productive work histories at the time of testing (as reported by their supervisors), suggesting that findings of HAD would be unlikely.

The mean age was $31.6( \pm 6.8)$, of which $23 \%$ were female and $77 \%$ male (which closely reflected the organisation's gender composition). Although the assessment was done in English, 98\% spoke eight other South African languages at home. In the group $8 \%$ had 10 to 11 years of formal education, $65 \%$ had completed high school, and $27 \%$ had a variety of post-school vocational training certificates.

\subsection{Assessment Data}

The NP battery was administered by clinical psychologists, in the order set out in Table 1. Norms that were stratified on demographic factors (age, gender) were applied where available; otherwise local SSA norms of broadly matched samples were used. Of the 60 cases, $75 \%$ were found without significant neurocognitive impairments, $20 \%$ were classified as ANI, and 5\% with MND. Due to the small numbers, ANI and MND were collapsed into a Mild Impairment category for statistical analysis. The descriptive statistics of the individual measures, and comparison of measures across outcomes, can be found in Table 2, which provides means and standard deviations, as well as comparisons using independent t-tests. The two groups did not differ significantly when using biographical markers (age, gender, language, or education), BRUMS scores, or CD4 count.

Table 2. Descriptive statistics of measures, and comparison across NP outcomes.

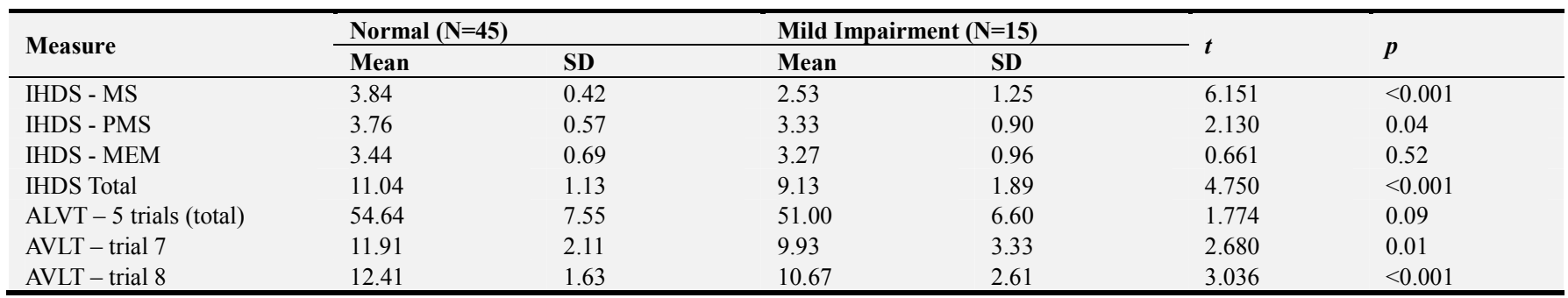




\begin{tabular}{|c|c|c|c|c|c|c|}
\hline \multirow{2}{*}{ Measure } & \multicolumn{2}{|c|}{ Normal $(N=45)$} & \multicolumn{2}{|c|}{ Mild Impairment $(\mathrm{N}=15)$} & \multirow[b]{2}{*}{$t$} & \multirow{2}{*}{$p$} \\
\hline & Mean & SD & Mean & SD & & \\
\hline AVLT - trial 9 & 14.61 & 0.75 & 14.07 & 1.22 & 2.051 & 0.04 \\
\hline TMT - trial A & 34.68 & 10.03 & 47.13 & 16.60 & -3.496 & $<0.001$ \\
\hline TMT - trial B & 72.18 & 19.27 & 120.91 & 54.74 & -5.120 & $<0.001$ \\
\hline $\mathrm{GP}-\mathrm{DH}$ & 60.83 & 8.04 & 73.27 & 18.61 & -3.626 & $<0.001$ \\
\hline $\mathrm{GP}-\mathrm{NDM}$ & 66.40 & 8.60 & 81.00 & 22.86 & -3.629 & $<0.01$ \\
\hline DSMT & 63.49 & 14.16 & 58.20 & 15.53 & 1.167 & 0.26 \\
\hline $\mathrm{DS}-\mathrm{F}$ & 9.82 & 2.35 & 9.67 & 2.02 & 0.240 & 0.81 \\
\hline DS - B & 5.91 & 2.27 & 4.80 & 2.57 & 1.486 & 0.15 \\
\hline
\end{tabular}

Seven markers differentiated between the normal and mild impairment groups, namely the IHDS Total score $(\mathrm{p}<.001)$, AVLT delayed recall $(\mathrm{p}<.001)$, TMT-A $(\mathrm{p}<.001)$ and B $(p<.001)$, GP $(p<.001$ for both DH \& NDH $)$, and TGT $(p<.001)$, suggesting that the BNCA may be useful in identifying individuals with potential problematic neurocognitive functioning. However, given the risk of circular logic, these findings need to be interpreted with caution.

In follow up to the BNCA, individuals with normal NP functioning were given general health counselling to maintain good NP health, while the 12 people classified with ANI were given health counselling appropriate to their respective situations. One person classified with MND was moved to a position with a lower personal risk profile prior to the assessment, and the other two initiated the same process after the assessment. All 60 continued to make positive contributions to the organisation.

\subsection{Discussion}

The composition of the battery was informed by seven guidelines, and the final product, for the most part, appears to adhere to it:

1. Observations by the test administrators indicated that it was both brief (less than $60 \mathrm{~min}$ to complete) and comprehensive enough to enable preliminary diagnostic classification.

2. Apart from the WAIS subtests and GP, it was inexpensive to obtain, and inexpensive to use on a cost-per-case basis.

3. Beside the GP (and a stopwatch), there was no special test equipment, making it easy to transport and set up.

4. For most of the tests/tasks, some form of comparable normative data was available, and though not always ideal, was in most cases sufficient for preliminary classification. However, more work is needed to establish acceptable comparable normative reference data across most of the tasks.

5. All the tests/tasks had a track record in the field of HAND testing, and directly tapped the prominent domains identified in the literature. Further, most of them were reported in studies from eastern and southern Africa, which has similar clade distribution as the population described in this paper [41].

6. Observations by the test administrators did not raise language as a significant issue in this educated population. However, language sensitivity remains a critical concern in NP testing in SSA, and needs further consideration even in higher educated samples.
7. Observations further suggested that technicians could be trained to administer the BNCA independently, allowing registered psychologists to focus on the interpretation of results.

The battery appeared - in this sample - to differentiate between persons with milder cognitive impairment, and those without. In many of the cases, high performance scores were recorded, possibly due to the educational attainment of those participants. Test administrators, in their written observations, reflected that some of the tasks (e.g. TMT-B) possibly did not provide enough of a challenge to elicit difficulties with either attention or executive function. A simple maze tracing task was included in the last few assessments, and may hold promise as an addition to the original battery. More demanding tasks - rather than simply more tests - may increase the battery's ability to differentiate between no impairment and mild impairment, and requires further development and testing. Further work is also required to establish tolerability of the BNCA process, as well as satisfaction of various role-players (patients, employers, etc.).

Workplace reports were available for most of the participants, but proved difficult to quantify - as correlates to NP test performance - and could not be used to enable meaningful associations between test scores and work performance. It is suggested that a scale for Activities of Daily Living be considered to supplement information on real-life behavioural indicators, although this may be more relevant to the more severe presentations of HAND. Further, given the prevalence of alcohol abuse (35\%) reported among HIV samples in SA [42], it may be useful to also include a screening tool for this prior to formal testing.

The particular process followed - as per the flowchart in Figure 1 -would prevent inappropriate testing, for example in cases where severe emotional distress or the inability to complete consent are present. This is a particular strength, as it can easily be facilitated by trained technicians or primary health care providers.

\section{Conclusions}

The impact of HAND at the workplace can be substantial, considering the prevalence of HAND in general clinical samples, as well as the potential for significant risks to the health and safety of individual workers and workgroups.

A brief-but-adequate-enough assessment such as the BNCA could be used to provide meaningful recommendations regarding both clinical management, as well as workplace 
utilisation, to employers. It can probably be implemented in most occupational contexts in SSA, on the condition that proper processes (e.g. conditions for assessments, appropriate follow up procedures, confidentiality, and so forth) are imposed and maintained.

In conclusion, this paper set out to describe a protocol for a brief neurocognitive assessment for HAND in low resourced occupational health settings, provided some data from an initial trial, and invites debate and discussion on any measures that could improve brief but comprehensive workplace assessments.

\section{Conflict of Interests}

The author declare that there are no conflicts of interest.

\section{References}

[1] Antinori A, Arendt G, Becker JT, et al. Updated research nosology for HIV-associated neurocognitive disorders. Neurol 2007; 69(18):1789-1799.

[2] Chan P, Brew BJ. HIV associated neurocognitive disorders in the modern antiviral treatment era: prevalence, characteristics, biomarkers, and effects of treatment. Curr HIV/AIDS Rep 2014; 11(3):317-24.

[3] Grant I. Neurocognitive disturbances in HIV. Int Rev Psychiatry 2008; 20:33-47.

[4] Grant I, Sacktor N, McArthur JC. HIV and neurocognitive disorders. In Gendelman HE, Grant I, Everall I, Lipton SA, Swindells S, eds. The Neurology of AIDS. $2^{\text {nd }}$ Edition. Oxford: Oxford University Press; 2005: 359-374.

[5] Heaton RK, Clifford DB, Franklin DR, et al. HIV-associated neurocognitive disorders persist in the era of potent antiretroviral therapy: CHARTER Study. Neurol 2010; 75(23):2087-96.

[6] Robertson KR, Smurzynski M, Parsons TD, et al. The prevalence and incidence of neurocognitive impairment in the HAART era. AIDS 2007; 21:1915-1921.

[7] Schouten J, Cinque P, Gisslen M, Reiss P, Portegies P. HIV-1 infection and cognitive impairment in the cART era: a review. AIDS 2011; 25:561-575.

[8] Simioni S, Cavassini M, Annoni JM, et al. Cognitive dysfunction in HIV patients despite long-standing suppression of viremia. AIDS 2010; 24:1243-1250.

[9] Joska JA, Westgarth-Taylor J, Myer L, et al. Characterization of HIV-associated neurocognitive disorders among individuals starting antiretroviral therapy in South Africa. AIDS Behav 2011; 15(6):1197-1203.

[10] Van Wijk CH, Meintjes WAJ. International HIV Dementia Scale: Screening for HIV associated neurocognitive disorders in occupational Settings. Occup Health Southern Afr 2015; 21(4):10-16.

[11] Dawes S, Suarez P, Casey CY, et al. Variable patterns of neuropsychological performance in HIV-1 infection. J Clin Exp Neuropsychol 2008; 30: 613-626.
[12] Cherner M, Cysique L, Heaton RK, et al. Neuropathologic confirmation of definitional criteria for human immunodeficiency virus-associated neurocognitive disorders. J Neurovirol 2007; 13:23-28.

[13] Robertson K, Liner J, Heaton R. Neuropsychological assessment if HIV infected populations in international settings. Neuropsychol Rev 2009; 19:232-249.

[14] Woods SP, Moore DJ, Weber E, Grant I. Cognitive Neuropsychology of HIV-Associated Neurocognitive Disorders. Neuropsychol Rev 2009; 19:152-168.

[15] Gorman AA, Foley JM, Ettenhofer ML, Hinkin CH, van Gorp WG. Functional consequences of HIV-associated neuropsychological impairment. Neuropsychol Rev 2009; 19:186-203.

[16] Bor J, Tanser F, Newell M-L, Bärnighausen T. In a study of a population cohort in South Africa, HIV patients on antiretrovirals had nearly full recovery of employment. Health Aff 2012; 31(7):1459-1469.

[17] Marcotte TD, Wolfson T, Rosenthal TJ, et al. A multimodal assessment of driving performance in HIV infection. Neurol 2004; 63:1417-1422.

[18] Mind Exchange Working Group. Assessment, Diagnosis, and Treatment of HIV-Associated Neurocognitive Disorder: A Consensus Report of the Mind Exchange Program. Clin Infect Dis 2013; 56:1004-1017.

[19] Watts AD, Shuttleworth-Edwards AB. Neuropsychology in South Africa: confronting the challenges of specialist practice in a culturally diverse developing country. Clin Neuropsychol 2016; 30(8):1305-1324.

[20] Thom RGM. HAART and Mind: Common Mental Disorders in People Living with HIV/AIDS. The Access Series. South Africa: Reach Publishers, 2012.

[21] Jonsson G, Davies N, Freeman C, et al. Management of mental health disorders in HIV-positive patients. S Afr J HIV Med 2013; 14(4):155-165.

[22] Haddow LJ, Floyd S, Copas A, Gilson RJC. A Systematic Review of the Screening Accuracy of the HIV Dementia Scale and International HIV Dementia Scale. PLoS ONE 2013; 8(4):e61826.

[23] Singh D, Sunpath H, John S, Eastham L, Gouden R. The utility of a rapid screening tool for depression and HIV dementia amongst patients with low CD4 counts - a preliminary report. Afr J Psychiatry 2008; 11:282-286.

[24] Singh D, Joska JA, Goodkin K, et al. Normative scores for a brief neuropsychological battery for the detection of HIV-associated neurocognitive disorder (HAND) among South Africans. BMC Res Notes 2010; 3:28.

[25] Joska JA, Westgarth-Taylor J, Hoare J, et al. Validity of the International HIV Dementia Scale in South Africa. AIDS Patient Care STDS 2011; 25:95-101.

[26] Stern Y, McDermott MP, Albert S, et al. Factors associated with incident Human Immunodeficiency Virus dementia. Arch Neurol 2001; 58:473-479.

[27] Carey CL, Woods SP, Rippeth JD, et al. Initial validation of a screening battery for the detection of HIV-associated cognitive impairment. Clin Neuropsychol 2004; 18:234-248 
[28] Sacktor NC, Wong M, Nakasujja N, et al. The International HIV Dementia Scale: a new rapid screening test for HIV dementia. AIDS 2005; 19:1367-1374.

[29] Yepthomi T, Paul R, Vallabhaneni S, et al. Neurocognitive consequences of HIV in southern India: A preliminary study of clade C virus. J Int Neuropsychol Soc 2006; 12:424-430.

[30] Maj M, D'Elia L, Satz P, et al. Evaluation of two new neuropsychological tests designed to minimize cultural bias in the assessment of HIV-1 seropositive persons: a WHO study. Arch Clin Neuropsychol 1993; 8:123-135.

[31] Lawler K, Mosepele M, Ratcliffe S, et al. Neurocognitive impairment among HIV positive individuals in Botswana: a pilot study. J Int AIDS Soc 2010; 13:15.

[32] Ferrett HL, Thomas KGF, Tapert SF, et al. The Cross-cultural Utility of Foreign- and Locally-derived Normative Data for Three WHO-endorsed Neuropsychological Tests for South African Adolescents. Metab Brain Dis 2014; 29(2):395-408.

[33] Army Individual Test Battery. Manual of directions and scoring. Washington, DC: War Department. Adjutant Generals Office, 1944.

[34] Andrews K, Shuttleworth-Edwards AB, Radloff SE. Normative indications for Xhosa speaking unskilled workers on the Trail Making and Stroop Tests. J Psychol Afr 2012; 22(3):333-342.
[35] Ruff RM, Parker SB. Gender and age-specific changes in motor speed and eye-hand coordination in adults: Normative values for the Finger Tapping and Grooved Pegboard Test. Percep Mot Skills 1993; 76:1219-1230.

[36] Van Wijk CH, Meintjes WAJ. Grooved pegboard for adult employed South Africans: Normative data and HIV associations. S Afr J Psychol 2015; 45(4):521-535.

[37] Holguin A, Banda M, Willen EJ, et al. HIV-1 Effects on Neuropsychological Performance in a Resource-Limited Country, Zambia. AIDS Behav 2011; 15:1895-1901.

[38] Wechsler D. Wechsler Adult Intelligence Scale. $3^{\text {rd }}$ Edition. New York: The Psychological Corporation; 1997.

[39] Price RW, Sidtis JJ. Evaluation of the AIDS dementia complex in clinical trials. J AIDS 1993; 3(Suppl 2):551-560.

[40] Robertson KR, Parsons TD, Sidtis JJ, et al. Timed Gait Test: Normative data for the assessment of the AIDS Dementia Complex. J Clin Exp Neuropsychol 2006; 28:1053-1064.

[41] Sacktor N, Nakasujja N, Roberson K, Clifford DB. HIV-associated cognitive impairment in sub-Saharan Africa the potential effect of clade diversity. Nat Clin Pract Neurol 2007; 3(8):436-443.

[42] Joska JA, Fincham DS, Stein DJ, Paul RH, Seedat S. Clinical correlates of HIV-associated neurocognitive disorders in South Africa. AIDS Behav 2010; 14(2):371-378. 